\title{
Erratum to: Clinical outcomes of tigecycline alone or in combination with other antimicrobial agents for the treatment of patients with healthcare-associated multidrug-resistant Acinetobacter baumannii infections
}

\author{
Y.-T. Lee • S.-M. Tsao ・ P.-R. Hsueh
}

Published online: 9 April 2014

(C) Springer-Verlag Berlin Heidelberg 2014

\section{Erratum to: Eur J Clin Microbiol Infect Dis} DOI 10.1007/s10096-013-1870-4

An error has occurred in the authors address. It should read:

Y.-T. Lee • S.-M. Tsao

Institute of Medicine, Chung Shan Medical University

Y.-T. Lee • S.-M. Tsao

School of Medicine, Chung Shan Medical University

The online version of the original article can be found at http:// dx.doi.org/10.1007/s10096-013-1870-4.

\section{Y.-T. Lee $\cdot$ S.-M. Tsao}

Institute of Medicine, Chung Shan Medical University, Taichung,

Taiwan

Y.-T. Lee $\cdot$ S.-M. Tsao

School of Medicine, Chung Shan Medical University, Taichung,

Taiwan

Y.-T. Lee $\cdot$ S.-M. Tsao

Division of Infectious Diseases, Department of Internal Medicine,

Chung Shan Medical University Hospital, Taichung, Taiwan

Present Address:

P.-R. Hsueh ( $\square)$

Departments of Laboratory Medicine and Internal Medicine,

National Taiwan University Hospital, National Taiwan University

College of Medicine, Taipei, Taiwan

e-mail: hsporen@ntu.edu.tw 